\title{
On Ancient China's Body and Spirit Conception and Its Modern Value --- Reference Analysis on Correlation between Network and Ideological Education of Adolescents
}

\author{
Tian Lu \\ Xi'an International University, Xi'an Shaanxi, 710000, China
}

Key words: Body and spirit conception, Modern value, Network, Ideological education of adolescents.

\begin{abstract}
Ancient China's body and spirit conception is rich in content, and the dialectical relationship it contains is a key to understanding the main body of human society and human spiritual world. With the constant development of network society, more and more social problems emerge, and the ideological education of adolescents even becomes dreadful. To solve this problem, the ancient body and spirit conception can be referred to for some perspectives and ideas of problem analysis, which reflects the modern value of the body and spirit conception.
\end{abstract}

\section{Introduction}

Since the pre-Qin period, the body and the spirit has been an important theoretical problem concerned in the realm of thought in China. The schools in the realm of thought hold their own viewpoints. The body and spirit conception is a proposition on the pair of body and spirit, relating to the correlation between human's spiritual phenomenon, attributes and cognitive ability and material carriers. In modern society, network has gradually develops into a spiritual world as a counterpart of social subject, especially for adolescents. We will develop the modern society by referring to the ancient China's body and spirit conception.

\section{"Body" and "Spirit" in China's Tradition}

"Body" literally refers to external and material things. People take it for granted that the "body" in the "the spirit is born from the formation of the body" refers to visible and touchable human body which consists of various solid substances constituting human body mentioned in physiological anatomy. In traditional philosophy, however, the "body" in the "the spirit is born from the formation of the body" refers to living body in motion, including both external static human body and internal organic whole system. Of course, the emphasis on organic characteristics of internal system doesn't mean denial of the characteristics of external entity of body. Internal organic characteristics and external entity characteristics stand for different aspects of the same problem. In China's traditional philosophy, "body" refers to living body of dynamic significance, namely every social individual, subject or group with thought, judgment ability and social thinking ability, as below.

What does "spirit" refer to? Immortality of soul is a concept based on which primitive man thought individual source, while primitive man thought clan source based on ancestor worship. Under the guidance of the idea of animism, people, as a token of appreciation and fear, worshipped natural objects by offering sacrifices and dancing, to get into good graces of gods for seeking happiness and avoiding calamity. This is the practice of primitive natural religion. Besides, myth and primitive religious fantasy were used to explain those unexplainable.

In a word, in ancient China's body and spirit conception, bedsides the common understanding of body and spirit, their concrete implications had been developed to a certain extent. "Body" doesn't 
only refer to the sensorial and material, but also to a dynamic system; "spirit" is not only born from "body", but also has its own connotation—-namely spirit place.

\section{Dialectical Relationship between Body and Spirit and Modern Value}

In the history of China, ideologists had drastic debates on what determined what, spiritual mortality or immortality, and belief in gods and ghosts and anti-superstition in relation thereto. Some held that "mentality or spirit is phenomenal existence, had neither energy nor mass, so it cannot drive or interfere brain or neural activities". [1] (PP.81 82) "The 'subjective initiative' we usually mention actually means subjective initiative of human brain, or some self-organizing function of nervous structure of brain; spiritual phenomenon depends on brain neurological motion to move. It cannot have real "initiative"." [1] (P.89) According to this viewpoint, spirit has neither mass nor energy, cannot have initiative, and cannot truly dominate the body, and it is the self-organizing function of nervous system that dominates the body.

As a matter of fact, those who hold this point didn't realize the self-organization of spirit. "Self-organization is an inherent attribute of all matters (including thinking system)", [2] (P.212) "even that the motor process of human spirits complies with the self-organization principle" [3] (P.269). The self-organization of spirit was produced on the basis of self-organization of nerve. Though emergence of self-organization of spirit was behind that of self-organization of nerve, the self-organization of spirit can determine and dominate the nervous system. The self-organization of the nervous system determines the initiative of spirit. Thus, denying the initiative of spirit means denying the self-organization of spirit and even of any system.

The analysis on correlation between body and spirit as above clearly shows that human was taken as an ordinary system in the universe in Chinese traditional philosophy, namely human consisting of body and spirit. The dialectical relationship between body and spirit actually is the dialectical relationship between the whole system and the part or elements. "The whole and the part is the precondition for interdependence and mutually succeeding of body and spirit. The whole consists the part or elements, and cannot exist without the part or elements. The part or elements are the part or elements only when they are a member of a system." [4] (P.131) Hence, that body and spirit depend on and succeed each other is the precondition. Body cannot stand alone without spirit, nor can spirit. It is in such a interdependency relation that body and spirit have obtained their own definitive properties. Though body is the basis for the production of spirit, spirit can determine and denominate body. The final formation of this viewpoint had lasted a long historical process.

We can observe the value of expositions and argumentations of ancient body and spirit conception to modern society by combining with modern society.

Firstly, a new schema-"body"-"spirit" relationship was put forward for analyzing and realizing subject-object relationship. Thus, we have a basis for analyzing some issues on subject-object relationship. This schema is a complete dialectical system.

Secondly, in this relational schema, the meaning of "body"-"spirit" relationship was interpreted in there ways, namely "body" being carrier of "spirit", and "spirit depending on "body" to exist; "spirit" being able to the existential state of "spirit" and even determine and denominate "spirit"; body and spirit being unified, which depend on and succeed each other.

Thirdly, body-spirit relationship is diversified and complex. In a modern sense, it is inevitable and proper to consider the complex society from multiple aspects. Thus, this schema enlightens us to overturn the original mode of thinking and consider the more diversified subject-object relationship from a new perspective.

A proper body and spirit conception is of great ideological and practical significance to criticizing some absurd heretical theory of the immortality or theism. Body and spirit are two elements of life body of human, and are an issue on the separation and reunion of body and spirit much concerned in philosophy, religion and natural science explorations. In particular, it is of greater significance in modern society, especially in the network society. 


\section{Network and Adolescent Education from the Perspective of Body and Spirit Conception}

On March 23, 2005 (Wednesday), a news story titled by Parents Scramble for An "Effective Prescription" of Internet Cessation" published in the $11^{\text {th }}$ page of the Hua Shang Daily reported a story that 500 disks provided by a professor for helping children to quit internet at a lecture jointly organized by several medias and middle schools were partitioned by parents less than two hours. The internet is not a new thing, but it still greatly attracts adolescents. Then, problems occur.

The emergence and expansion of network and the networking tendency have integrated science and technology, and connect the group, organization, society and culture of human, having greatly extending the boundaries of time and space of survival and development of human. As the relationship between human and network becomes closer and closer, the netizen group take on a younger-age trend. Adolescence is a key period when a person develop and form his morality. The network society greatly affects adolescents' moral judgment and moral behavior, and even the basic value composition of their spiritual world. As a whole, adolescents are characterized by strong curiosity, emulation, high vigorousness, eager for rich knowledge, and need of equal communication. The internet is attracting adolescents with its peculiarity, novelty and mystery. A survey shows that the age group of netizens ranging from 25 to 30 accounts for $74.59 \%$, and the group of which the educational level reaches junior college and above for 70.11\%. [5] (P2) The inventor of the internet asserted: the internet is a "free and equal", where there is no "government, police, army, class, poverty, and discrimination". The colorful cyber world, however, is also like Pandora's Box despite of its huge social function. This poses a severe challenge to ideological education of adolescents. From this perspective, the cyber world is a spiritual world, at least acting as a counterpart of the collection of social individuals who are the subject of the society. Thus, the "body"-"spirit" subject-object relationship schema is established.

Subject and object are a pair of category that philosophers have been discussing about. The philosophers in each period will concern the status of subject in their period. Subject refers to human who carry out practice and recognition activities, while object to the objects of practice and recognition activities. In practice, the subject objectifies the essential power, transforms the object, and internalize the attributes and laws of the object to be its essence to enrich and develop its essential power. Subjectivity reflects the status of the object and the way it relates to the object. The fundamental forms of human activities include production practice and recognition activity, in which the value relation and aesthetic relation between human and the object run through. Accordingly, human act as practical subject, cognitive subject, value subject or aesthetic subject in different activities. The subject refers to the subject in subject-object relationship, specifically, to human, and the object refers to the world. Human can be taken as individual or group. The world consists of two parts: external things of specific human, and man himself. External things include natural objects, social existence, other people, etc.; man himself is the object that man reflects on, which shows the subjectivity of man. In the human-nature, human-society and human-human relationships, the subjectivity of man is realized via man's practice and recognition activity. The human-internet relationship becomes a new relationship, in which the object of practice and recognition activity exists in a networked way, and affects the existence situation of human who is the subject. The internet gradually develops into a "spirit" to stand as a counterpart of the "body" of social subject, and even override and dominate the subject. Besides, adolescents in the social subject are vulnerable to the internet. Here comes a crisis.

The internet "is not only an information source; it is a way in which people perform self-organization". [6] (P52) Though the internet is widely regarded as an important educational tool, a survey in California shows that $86 \%$ primary and middle school teachers thought the internet didn't work to improve students' school performance. Another survey shows that $58 \%$ students of a university in Pennsylvania underperformed in learning for they spent too much time on the internet. A psychologist of University of Texas at Austin found that at least 14\% students reached the level of internet addition disorder. Doctor Kendall of the Counseling Center of University of Maryland organized a university-wide mutual-help group to help those addicted themselves in the internet 
immediately after conducting a survey over the students of the university. The internet-related things most influential to male students are pornographic websites and computer games, while that to female students are chatting software and entertainment. A survey shows that those most vulnerable to internet addiction disorder are middle-aged housewives and college students in America; male internet addicts around 30 years old with college education background and above take a large proportion in Switzerland. In Britain, a survey made by the British Psychology Society shows that the main netizen group are well-educated introvert people at the age ranging from 20 to 30 . There is no related data about China, but it can be foreseen that the netizen group mainly consist of well-educated adolescents, and the body-spirit is changing

1."Body"-"spirit" transformation is a warning of danger. The boundary between the cyber world and the spiritual world of adolescents is fuzzy. As mentioned above, the "body" as subject is an immature dynamic system. Thus, what will happen in the process of receiving "spirit" is uncertain. They communicate fast, have few choices, are good at imitating, and are popular, so that they confuse their living world with the network society. This has become a contest between "spirit" and "spirit". Obviously, the latter is weaker.

2. The internet, as informationized physical extension of the spirit of the subject (adolescents), has become aggressive, which erodes the "spirit" of the subject. "Spirit" has become a spirit place and a place where spirit is born. Such aggressivity of spirit usually incubates in the ideology of adolescents, which may burst out under a strong stimulation at a time, and cause severe social consequences. This latency comes from the unconscious inner driving force of harmful information such as cyber-violence, eroticism, nonsense, etc., and the inner driving force is transmitted in such a way of "network spirit"-"subject spirit"- "subject body".

3. Thus the informationized physical extension of the spirit becomes another kind of "spirit" and coexists with the original "spirit" of the subject, becoming the third kind of objectified spirit or "informationized spirit" and "network spirit" born in the spiritual world of the subject and the cyber world. It is the third kind of "spirit" born by human body and network together.

To sum up, it is of great reference value to analyze social problems based on ancient body and spirit conception, especially network and ideological education of adolescents. If deep studies are conducted, it may be found that these problems do not only relate to adolescents, but to the whole society and even the whole world. Of course, we don't reject modern technology (especially network), but hope the modern society to integrate more humanistic and ecological ideas that are more suitable for it, to continue the world healthily.

\section{References}

[1] Wei Zhi: On Ego, Beijing: Wenjin Press, 1991.

[2] Zhan Kenhua: Self-organization and System Evolution, Social Sciences in China, 1986, (6).

[3] Wei Hongsen, Zeng Guoping: System Theory-Philosophy of System Science, Beijing: Tsinghua University Press, 1995.

[4] Xiao Ming: Philosophy,Beijing: Economic Science Publishing House, 1991.

[5] China Youth, Edition 6, 2001

[6] (America) Esther Dyson: A Design for Living in the Digital Age, Hainan Publishing House Printed in 1998. 